\title{
PROJECTIONS AND RETRACTIONS
}

\author{
JOHN B. CONWAY
}

The main result of this paper is a generalization of a theorem of R. S. Phillips [6] on the nonexistence of projections of $l^{\infty}$ onto $c_{0}$. If $S$ is a locally compact Hausdorff space then let $C(S)$ denote the space of bounded continuous real (or complex) valued functions on $S$; also, let $C_{0}(S)$ be those functions in $C(S)$ which vanish at infinity. If $N$ is the space of positive integers with the discrete topology then $l^{\infty}=C(N)$ and $c_{0}=C_{0}(N)$. Thus, Phillips' theorem says that in the case where $S=N$ there is no bounded projection of $C(S)$ on to $C_{0}(S)$; that is, the space $N$ does not have the projection property.

It is natural to ask for a characterization of spaces with the projection property in terms of their topology. Unfortunately, we cannot achieve this but we do show in Theorem 2 that pseudocompactness is a necessary condition for this property ( $S$ is pseudocompact if and only if every real valued continuous function on $S$ is bounded). However, as Example 3 demonstrates, pseudocompactness is not sufficient.

As a corollary to Theorem 2 we obtain a result of $\mathrm{W}$. W. Comfort [1]. Namely, if $S$ is completely regular and there is a retraction of its Stone-Čech compactification $\beta S$ onto $\beta S \backslash S$ then $S$ is a locally compact peudocompact space. To establish his result Comfort appealed to a result of W. Rudin which depends on the continuum hypothesis. Hence, not only do we give a relatively simple proof of Comfort's theorem, but furthermore, we do so without using the continuum hypothesis.

Before proceeding to the main theorem we will need the following theorem of I. Glicksberg [3]. The proof is not difficult and we shall not repeat it here.

Theorem 1. A completely regular Hausdorff space $S$ is pseudocompact if and only if for every sequence $\left\{V_{n}\right\}$ of nonvoid open sets with pairwise disjoint closures there is an $s_{0} \in S$ such that for every integer $n_{0}$ and every open neighborhood $V_{0}$ of $s_{0}$ there is an $n>n_{0}$ with $V_{0} \cap V_{n}^{-}$ $\neq \square$.

We can now prove our main theorem.

THEOREM 2. Let $S$ be a locally compact Hausdorff space; if there is a bounded projection of $C(S)$ onto $C_{0}(S)$ then $S$ is pseudocompact.

Received by the editors October 15, 1965. 
Proof. Let $P: C(S) \rightarrow C_{0}(S)$ be the hypothesized projection and suppose $S$ is not pseudocompact. By Theorem 1 we can find a sequence $\left\{V_{n}\right\}$ of nonvoid open sets with pairwise disjoint closures such that $\left\{V_{n}^{-}\right\}$has no cluster points; i.e., $\bigcup_{n=1}^{\infty} V_{n}^{-}$is closed.

For every integer $n \geqq 1$ let $s_{n} \in V_{n}$ and choose a neighborhood $U_{n}$ of $s_{n}$ such that $U_{n}^{-}$is compact and contained in $V_{n}$. Using Urysohn's lemma we may find a function $\phi_{n}$ in $C(S)$ with $0 \leqq \phi_{n} \leqq 1, \phi_{n}\left(s_{n}\right)=1$, and $\phi_{n}(s)=0$ for $s \in U_{n}$. Let $F=\cup_{n+1}^{\infty} U_{n}^{-}$; then $s \in F^{-} \subset \cup_{n=1}^{\infty} V_{n}^{-}$implies that $s \in V_{n}^{-}$for a unique $n$. It easily follows that $s \in U_{n}^{-}$, and so $F$ is closed.

If $\xi=\left\{x^{(n)}\right\}_{n=1}^{\infty} \in l^{\infty}$ then we define

$$
f_{\xi}(s)=\sum_{n=1}^{\infty} x^{(n)} \phi_{n}(s)
$$

for all $s \in S$. Since the sets $\left\{V_{n}^{-}\right\}$are pairwise disjoint and each $\phi_{n}$ vanishes off $V_{n}^{-}$, we have that at most one term in the sum is not zero and so $f_{\xi}$ is well defined. For this same reason and because each $\phi_{n}$ achieves its supremum, $\left\|f_{\xi}\right\|_{\infty}=\|\xi\|_{\infty}$. To see that $f_{\xi}$ is continuous let $\left\{s_{i}\right\}$ be a net in $S$ which converges to a point $s \in S$. If $s \in F$ then $\left\{s_{i}\right\}$ is eventually outside of $F$ because $F$ is closed. Hence, there is an $i_{0}$ such that for $i \geqq i_{0}, f_{\xi}\left(s_{i}\right)=f_{\xi}(s)=0$ and so $\left\{f_{\xi}\left(s_{i}\right)\right\}$ converges to $f_{\xi}(s)$. If $s \in F$ then $s \in U_{n}^{-} \subset V_{n}$ for a unique integer $n$. Thus, there is an $i_{0}$ such that for $i \geqq i_{0}, s_{i} \in V_{n}$. But $s_{i} \in V_{n}$ implies $f_{\xi}\left(s_{i}\right)=x^{(n)} \phi_{n}\left(s_{i}\right)$ and so $\left\{f_{\xi}\left(s_{i}\right)\right\}$ converges to $f_{\xi}(s)$. Therefore $f_{\xi} \in C(S)$.

Define the linear transformation $T_{1}: l^{\infty} \rightarrow C(S)$ by $T_{1} \xi=f_{\xi}$; then $T_{1}$ is an isometry. Furthermore, if $\xi \in c_{0}$ then $T_{1} \xi \in C_{0}(S)$.

If $\phi \in C_{0}(S)$ and $\epsilon>0$ then $K=\{s:|\phi(s)| \geqq \epsilon\}$ is compact, and so $K \cap U_{d}^{-} \neq \square$ for at most a finite number of $U_{n}^{-}$. Let $n_{0}$ be such that for $n \geqq n_{0}, K \cap U_{n}^{-}=\square$. Thus, if $n \geqq n_{0},\left|\phi\left(s_{n}\right)\right|<\epsilon$; i.e., $\left\{\phi\left(s_{n}\right)\right\}_{n=1}^{\infty}$ is in $c_{0}$. Define $T_{2}: C_{0}(S) \rightarrow c_{0}$ by $T_{2} \phi=\left\{\phi\left(s_{n}\right)\right\}$; then $T_{2}$ is linear and bounded.

From these facts we have that $\pi: l^{\infty} \rightarrow c_{0}$ defined by $\pi=T_{2} \circ P \circ T_{1}$ is a bounded linear transformation. But $\xi \in c_{0}$ implies $T_{1} \xi \in C_{0}(S)$ and, since $P$ is a projection on to $C_{0}(S)$, we have $P\left(T_{1} \xi\right)=T_{1} \xi$. Moreover, since $\phi_{n}\left(s_{n}\right)=1$ for all $n$, we have that $\pi \xi=T_{2}\left(T_{1} \xi\right)=\xi$ and $\pi$ is a projection of $l^{\infty}$ onto $c_{0}$. This contradicts the result of Phillips and so $S$ must be pseudocompact. This completes the proof.

To prove Comfort's theorem we will need the following easy lemma.

Lemma. Let $X$ be a compact Hausdorff space and $S$ an open subset of $X$; if $f \in C(X)$ then let $f_{S}$ be the restriction of $f$ to $S$. Then the mapping which takes each $f$ into $f_{S}$ defines an isometric isomorphism between 
$C_{0}(S)$ and the subspace of $C(X)$ consisting of all functions vanishing on $X \backslash S$.

COROLlARY (COMFORT). If $S$ is a completely regular Hausdorff space and there is a retraction $r$ of $\beta S$ onto $\beta S \backslash S$ then $S$ is locally compact pseudocompact.

Proof. Since $\beta S \backslash S=r(\beta S)$ we have that $S$ is open in $\beta S$ and hence locally compact. Define $P_{1}: C(\beta S) \rightarrow C(\beta S)$ by $P_{1}^{2} f=f \circ r$; then $P_{1}$ is a bounded linear transformation. Also $P_{1}^{2} f=P_{1}(f \circ r)=(f \circ r) \circ r=f \circ r$ $=P_{1} f$ so that $P_{1}$ is a projection. Finally, $P_{1} f=0$ if and only if $f(\beta S \backslash S)$ $=0$. By the lemma we have that $P_{1} f=0$ if and only if the restriction of $f$ to $S$ belongs to $C_{0}(S)$. Hence, if $i: C(S) \rightarrow C(\beta S)$ is the map which takes each function in $C(S)$ onto its unique extension to $\beta S$, then $P=i^{-1} \circ P_{1} \circ i$ is a projection on $C(S)$ with $C_{0}(S)$ as kernel. Therefore $I-P$ is a projection of $C(S)$ onto $C_{0}(S)$ and, by Theorem $2, S$ must be pseudocompact. This completes the proof.

We say that $S$ has the retraction property if and only if there is a retraction of $\beta S$ on to $\beta S \backslash S$. Thus, by the preceding corollary, the retraction property implies the projection property, which in turn implies pseudocompactness. That the reverse implications are false will be illustrated by some examples. Moreover, the projection property is preserved by taking finite Cartesian products while the retraction property is not [1].

THEOREM 3. If $S_{1}$ and $S_{2}$ are locally compact spaces which have the projection property then so does $S_{1} \times S_{2}$.

Proof. By Theorem 2, $S_{1}$ and $S_{2}$ are pseudocompact; since they are also locally compact, it follows from a result of Glicksberg [4] that $S_{1} \times S_{2}$ is pseudocompact and $\beta\left(S_{1} \times S_{2}\right)=\beta S_{1} \times \beta S_{2}$. If $P_{i}$ : $C\left(\beta S_{i}\right) \rightarrow C\left(\beta S_{i}\right)$ is a projection with image $C_{0}\left(S_{i}\right), i=1,2$ (for notational reasons we will identify $C_{0}\left(S_{i}\right)$ with the space of functions in $C\left(\beta S_{i}\right)$ which vanish on $\left.\beta S_{i} \backslash S_{i}\right)$, then define

$$
P_{1} \otimes P_{2}: C\left(\beta S_{1}\right) \otimes C\left(\beta S_{2}\right) \rightarrow C\left(\beta S_{1} \times \beta S_{2}\right)
$$

by

$$
P_{1} \otimes P_{2}\left(\sum_{i=1}^{n} f_{i} \otimes g_{i}\right)=\sum_{i=1}^{n}\left(P_{1} f_{i}\right)\left(P_{2} g_{i}\right)
$$

where $f_{i} \in C\left(\beta S_{1}\right), g_{i} \in C\left(\beta S_{2}\right)$ for $1 \leqq i \leqq n$. Then $P_{1} \otimes P_{2}$ is a well defined linear map; furthermore $P_{1} \otimes P_{2}$ is continuous if $C\left(\beta S_{1}\right) \otimes C\left(\beta S_{2}\right)$ has its bi-equicontinuous topology (which is here a norm topology) and $C\left(\beta S_{1} \times \beta S_{2}\right)$ has its supremum norm (see [5, pp. 89-93] for 
most of the results needed here). But $C\left(\beta S_{1} \times \beta S_{2}\right)=C\left(\beta S_{1}\right) \otimes_{\lambda} C\left(\beta S_{2}\right)$, the completion of $C\left(\beta S_{1}\right) \otimes C\left(\beta S_{2}\right)$ in the bi-equicontinuous norm. Therefore $P_{1} \otimes P_{2}$ can be extended to a bounded linear transformation

$$
P: C\left(\beta S_{1} \times \beta S_{2}\right) \rightarrow C\left(\beta S_{1} \times \beta S_{2}\right) .
$$

Now, $\left(P_{1} \otimes P_{2}\right)^{2}=P_{1} \otimes P_{2}$ implies $P$ is a projection and hence has a closed image. But the image of $P_{1} \otimes P_{2}$ is the set of all functions of the form $\sum \phi_{i} \psi_{i}$ where $\phi_{i} \in C_{0}\left(S_{1}\right)$ and $\psi_{i} \in C_{0}\left(S_{2}\right)$. Hence, the image of $P_{1} \otimes P_{2}$ is dense in $C_{0}\left(S_{1} \times S_{2}\right)$ which implies that $C_{0}\left(S_{1} \times S_{2}\right)$ is the image of $P$. Therefore $S_{1} \times S_{2}$ has the projection property and the proof is complete.

Examples. (1) If $\Omega_{0}$ is the space of ordinal numbers less than the first uncountable ordinal $\Omega$ with the order topology, then $\beta \Omega_{0}$ $=\Omega_{0} \cup\{\Omega\}$, the one-point compactification of $\Omega_{0}$ [2, p. 75]. Clearly $\Omega_{0}$ has the retraction property and hence the projection property. Comfort [1] showed that $\Omega_{0} \times \Omega_{0}$ does not have the retraction property, but by Theorem 3, it does have the projection property. A more simple example of such a space is the following.

(2) Let $L_{1}, L_{2}$ be two long lines; i.e., $L_{1}$ and $L_{2}$ are the spaces $\Omega_{0} \times[0,1)$ with the order topology obtained from the lexicographic ordering. Let $L$ be the quotient space gotten by identifying the first points of $L_{1}$ and $L_{2}$. Then $L$ is a "long line which is long in two directions." Then $\beta L=L \cup\{\Omega,-\Omega\}$ and let the closure of $L_{1}$ in $\beta L$ equal $L_{1} \cup\{\Omega\}=\beta L_{1}$. As Comfort points out, $L$ does not have the retraction property since $\beta L$ is connected and $\beta L \backslash L$ consists of two points. then

However, if $g \in C(\beta L)$ such that $0 \leqq g \leqq 1, g\left(L_{2}\right)=0$ and $g(\Omega)=1$

$$
P f=f-[f(\Omega)-f(-\Omega)] g-f(-\Omega)
$$

is a bounded projection on to $C_{0}(L)$.

(3) We will now give an example of a pseudocompact space which does not have the projection property. Let $\Lambda=\beta R \backslash(\beta N \backslash N)$ (see $6 p$ of [2]). ${ }^{1}$ Then $\Lambda$ is pseudocompact and $\beta \Lambda=\beta R$. Let $\phi_{n} \in C(R)$ such that $0 \leqq \phi_{n} \leqq 1, \phi_{n}(n)=1$, and $\phi_{n}(s)=0$ for $|s-n| \geqq 1 / 3$. Then, as in the proof of Theorem 2, for every $\xi=\left\{x^{(n)}\right\}$ in $l^{\infty}$,

$$
f_{\xi}=\sum_{n=1}^{\infty} x^{(n)} \quad \phi_{n} \text { is in } C(R) .
$$

1 We would like to thank Dr. Stelios Negrepontis for calling our attention to this space. 
Hence $f_{\xi}$ has an extension to $\beta R$. But $\beta R=\beta \Lambda$ implies that $T_{1}$ : $l^{\infty} \rightarrow C(\Lambda)$ defined by $T_{1} \xi=f_{\xi}$ is a linear isometry. Also $T_{2}: C(\Lambda) \rightarrow l^{\infty}$ defined by $T_{2} f=\{f(n)\}$ is a bounded linear transformation. Therefore, if there is a bounded projection $P$ of $C(\Lambda)$ onto $C_{0}(\Lambda)$ then $T_{2} \circ P \circ T_{1}$ is a bounded projection of $l^{\infty}$ onto $c_{0}$, contradicting the theorem of Phillips.

Let us close by pointing out that if $S$ is a locally compact nonpseudocompact space then Theorem 2 says there is no simultaneous extension of $C(\beta S \backslash S)$ into $C(\beta S)$ (see [7] for details).

\section{REFERENCES}

1. W. W. Comfort, Retractions and other continuous maps from $\beta X$ onto $\beta X \backslash X$, Trans. Amer. Math. Soc. 114 (1965), 1-9.

2. L. Gillman and M. Jerison, Rings of continuous functions, Van Nostrand, Princeton, N. J., 1960.

3. I. Glicksberg, The representation of functionals by integrals, Duke Math. J. 19 (1952), 253-261.

4. - Stone-Čech compactifications of products, Trans. Amer. Math. Soc. 90 (1959), 369-382.

5. A. Grothendieck, Produits tensoriels topologiques et éspaces nucléaires, Mem. Amer. Math. Soc. No. 16, Amer. Math. Soc., Providence, R. I., 1955.

6. R. S. Phillips, On linear transformations, Trans. Amer. Math. Soc. 48 (1940), 516-541.

7. Z. Semadeni, Isomorphic properties of Banach spaces of continuous functions, Studia Math. (Seria Specjalna) 1 (1963), 93-108.

INDIANA UNIVERSITY 